Bull's seven and Le Bec's nine cases seem to have been a matter of serious discouragement to these operators. Bull says in the article already quoted: "This fatal result is worthy of notice in so far as it serves to emphasize my conviction that old and feeble subjects should not be subject to operation in reducible cases."

Le Bec says in his letter: "I dislike to operate for the radical cure of hernia in the aged as it is scarcely necessary in those who do not labor and the results are doubtful." His nine cures to one death, and that from pneumonia fourteen days after the operation, the peritoneum being normal, should be regarded more favorably.

Mr. Edmund Owen says "Age is an indefinite term and no barrier to the performance of an otherwise successful operation."

N. Senn says: "I have operated on a number of persons over 60 years of age for radical cure of hernia, usually after operation for strangulation. Age did not seem to interfere with a good result. The last patient was 78 years old. Strangulation of sigmoid flexure. Complete recovery in four weeks."

In reviewing the table and giving attention especially to the experience of H. O. Marcy, G. Stanmore Bishop, W. T. Bull, Le Bec, Arthur T. Barker, Aug. Broca, J. D. Griffith, W. W. Keen and Jos. Ransohoff, it would seem to me that precedent was established and prognosis sufficiently favorable to warrant us in recommending the operation to the aged in suitable cases as a matter of increased safety and comfort to those in whom reduction is impossible or retention difficult.

NoTE. - Recent experience with formalin catgut leads me to believe that it may entirely supplant kangaroo tendons in hernia operations. An autopsy one month after an exploratory laparotomy in a case of cancer of the stomach, where the peritoneum had been closed with a continuous suture of fine formalin catgut, showed it still firm enough to maintain support, but undergoing absorption. Previous to the appearance of Professor Șenn's article (See Journal of THe American Medical AssoCIATION for Dec. 12,1896 , p. 1219) I had exposed catgut for one week, instead of twenty-four hours, to the formalin solution. Before boiling it is brittle, but after boiling has great strength. It requires a longer time for absorption, as I have proved in frequent trials of both methods.

\section{ABSCESS OF THE SEPTUM NARIUM.} BY W. E. CASSELBERRY, M.D.

\section{CHICAGO, ILL.}

Chairman of the Section on Laryngology and Otology of the American Association: Professor of Laryngology and Rhinology in Northwestern University, Iredical school; Laryngologist and Rhinologist to st. Luke's Hospital;

Laryngologist to Wesley Hospital, etc.

The condition which, for the sake of simplicity in nomenclature is designated "Abscess of the Septum," only occasionally presents the typical characteristics of acute circumscribed suppuration. When caused by traumatism the first stage may be an effusion of blood beneath the muco-perichondrium of the cartilaginous septum which is termed "Hematoma of the Septum." In time the blood degenerates, perhaps becomes infected by pyogenic microörganisms and changes to a brownish yellow fluid which when evacuated appears neither like blood nor typical pus. In this state it has been called " Cold Abscess," and again when it does originate without traumatism and runs an acute course with pain, fever and obstructive swelling terminating in ideal suppuration, it has been spoken of as "Acute Perichondritis." Jurasz" has described a form in which the contained fluid is at first serous, termed serous perichondritis. Some sort of abscess is the culminating stage of all these conditions.
Although it might be possible for the bony portions to be affected, the usual seat of the disease is the cartilaginous part of the septum and it may be either unilateral or bilateral.

Etiology.-The most frequent cause is believed to be traumatism, e.g., blows or falls upon the nose, yet in many cases it is impossible to establish this relationship; slight contusions, readily forgotten, can determine a hematoma of the septum. This may run an insidious course, endure for days or weeks and undergo spontaneous absorption, having been little noticed, but in other instances the blood changes to sanguinolent pus and the cartilage itself disintegrates, the contents being retained by the bulging mucoperichondrium. Pyogenic microörganisms could excite suppuration in these and also in so-called idiopathic cases by gaining an entrance through excoriations which frequent these surfaces. The disease may occur also during the course of typhoid fever or smallpox, and Scheck calls attention to the frequency with which facial erysipelas proceeds from abrasions upon the septum and infers that abscess of the septum can be caused by infection by the streptococcus erysipelatis. In many cases still it is quite impossible to assign any definite cause.

This was true in the following case which is a typical example of the variety termed cold abscess.

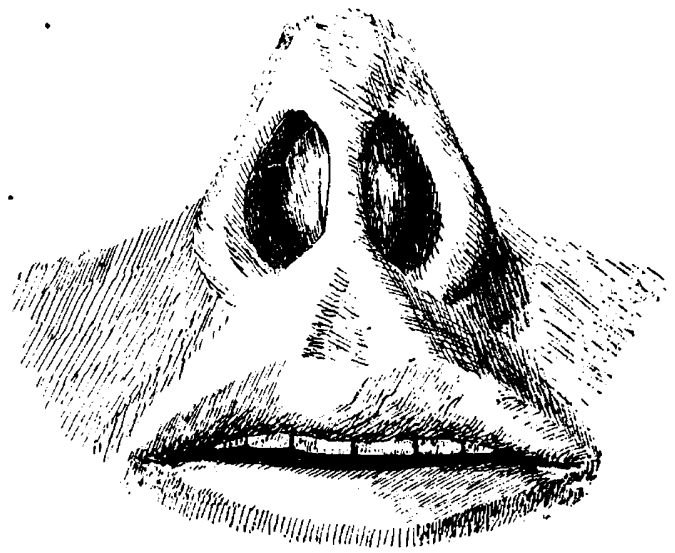

Case 1.-Master G., aged 16 years; while away from home at school began to suffer from nasal obstruction which he attributed to an acute exacerbation of his customary "catarrh." No history of traumatism could be elicited, the disease having seemingly commenced as a cold and continued several weeks before it received serious consideration. But nasal obstruction, and swelling within and without the nose gradually grew so serious that he was sent home where he was confined to his bed, supposed to be suffering from asthma. On examination the diagnosis was at once apparent, for from each side of the septum bulged a fluctuating tumor which completely blocked both nostrils. The enveloping mucosa was unbroken, somewhat inflamed, and the nose generally reddened and edematous. A brownish yellow liquid was withdrawn by a hypodermic syringe, and this was followed by a free incision toward the base of one side and gentle curetting of the cavity. The central portion of the cartilage had liquified, but it redeveloped from the muco-perichondrium and perfect recovery ensued.

Symptoms.-In case of the transition of a hematoma into an abscess, the disease may manifest itself either quite insidiously or the suppurative change may ensue quickly and be associated with sneezing, nasal irritation and slight fever. In either event the salient symptom ultimately complained of will be obstruction to nasal respiration together with the discomforts of mouth breathing.

When the abscess arises in the form and in conse-

Journal of Laryngology, etc., Nov. 1896, p. 266. 
quence of acute perichondritis it is ushered in during a few days by symptoms indicating a high degree of inflammation, $e \cdot g$, chill, pronounced fever, pain, swelling and redness of the whole organ. Spontaneous rupture is more apt to occur quickly in this than in the former type, although it may be much delayed after the subsidence of the inflammatory symptoms, leaving again nasal obstruction as the salient symptom.

Case 2 exemplifies the latter type.-Mr. B., aged 26 years; thought he had contracted a severe "cold in the head," the condition commencing with chilly sensations followed by head ache, fever, local sensitiveness and redness extending to the bridge of the nose. These symptoms gradually subsided but were replaced by nasal obstruction and what he now supposed was chronic nasal catarrh. The examination was not made till the third week when a fluctuating tumor was observed to bulge from each side of the septum. Typical pus was evacuated by an incision and the cavity curetted. The destroyed central part of the cartilage ultimately redeveloped without deformity. $\mathrm{He}$ positively denied the least probability of traumatism as a cause.

Diagnosis.-By simple inspection with the head tilted backward, the semicircular tumor may be seen bulging from one or both sides of the septum. Palpation by a probe will cause deep pitting or even fluctuation, and aspiration by a hypodermic needle will provide a sample of the contents. It may thus be readily distinguished from polypus for which it is most commonly mistaken by novices, but which occurs but rarely in this situation, and also from syphilitic gumma which commonly develops at just this spot. I have seen one case of gumma in which the central part had liquified before the occurrence of superficial ulceration, in which the diagnosis would have been impossible, had not other syphilitic signs been present.

Prognosis.- If recognized reasonably early and the abscess evacuated, the prognosis is very good. Notwithstanding complete liquefaction of the cartilage itself, if the muco-perichondrium be preserved, snother cartilaginous septum will develop and this usually without deformity. However, if the disease be unrecognized or if the inflammatory action extend to the antero-superior border of the septum, the line of junction of the septum with the lateral cartilage and nasal bones, softening and depression of these which constitute the dorsum of the nose will result. Apart from this "saddle-back" deformity or in conjunction with it, perforation of the septum can also ensue.

Treatment.-When the initial stage is a hematoma cold applications would be suitable. In any event as soon as pus is present a rather free incision should be made low down or one side and the contents expressed. This should be reopened daily with a probe until the cavity has been effaced. It is probably not necessary to curette, wash out or pack the cavity with gauze although one or all of these may seem desirable at times.

\section{FIBROUS NASAL POLYPUS.}

\section{BY D. R. SILVER, M.D.} SIDNEY, OHIO.

As a contribution to our knowledge of nasal polypi, I venture to report this case, which, in one respect at least, may be unique. The growth developed in a boy, 12 years old. He had the characteristic mouthbreathing at all times on account of the complete closure of the posterior nares. On inspection, a tumor the size of a man's thumb could be seen in the naso- pharynx below the line of the soft palate. To the touch it was hard and seemed firmly attached; so firmly, indeed, that to remove it by twisting was out of the question. Its point of attachment could not be made out. Its large size made the passage of anything through either nostril nearly impossible. As a preliminary step, a threaded needle was passed through the lower portion of the growth so that it could be pulled well down and forward, after which it was found possible to pass a small rubber catheter, to which was attached a string, through the right nostril and out of the mouth, as in the operation for closing the posterior nares. By means of the string a heavy silver wire, doubled, was drawn through and slipped over the tumor, fortunately encircling the point of attachment, which could hardly be called a pedicle, since it was found to be nearly as large as any other portion of the neoplasm, and fully threefourths of an inch in diameter. The wire attached to a strong screw cut its way through in an hour.

It proved to be a three-lobed affair, very hard and fibrous, and was drawn out through the mouth by means of the thread which transfixed the growth at its lower end. Its dimensions were $2 \frac{3}{4}$ by $1 \frac{1}{3}$ by $\frac{3}{4}$ inches. The pain produced by the constricting wire was considerable, but there was no hemorrhage to occasion alarm. Notwithstanding the fact that the point of attachment was well cauterized a large mucous polypus sprang up in a few weeks. This was removed by twisting and did not return. The specialist trained in the manipulations of rhinologic work may not find the removal of nasal and post-nasal polypi very difficult. But when these growths are very large or very small the general practitioner finds their management quite perplexing. For this reason the subjects of nasal polypi furnish a large contingent of the army of victims who fall an easy prey to the catarrh "specialist." Nostrums, whose only recommendation is their nastiness, are bought and used for years by persons whose rather fashionable complaint could be removed by a twist of the forceps.

\section{HEMORRHAGE FROM THE LARYNX, FOL- LOWING AN ATTACK OF MEASLES, IN AN ADULT.}

Read before the Section of Otology and Laryngology of the College of Physicians, Philadelphia, Pa.. March 3, 1896.

BY FRANK WOODBURY, M.D.

Instructor in Laryngology in the Philadelphia Polyclinic; Attending Physician to the Medico-Chirurgical Hospital, etc. PHILA DELPHIA, PA.

On January 24, Martin S., 27 years of age, born in Ireland, a laborer by occupation, presented himself at the laryngologic dispensary of the Polyclinie Hospital with an acute inflammation of the fauces and pharynx and with almost extinguished voice, requiring considerable effort in speaking. He gave the following history: About nine weeks previous, or about the first of December, four sailors were admitted to the Naval Hospital suffering with measles. Three cases developed subsequently among the employes of the institution, where he was engaged as a fireman, and all the cases recovered. The patient was taken sick January 3, or about two weeks after the first patients were convalescent, and had been allowed to go around the grounds. He said that he had fever, eruption, conjunctivitis, cough, and subsequently there was much desquamation, especially from his face 